\title{
Razas y Gama de Hospedantes en Diferentes Poblaciones del Nematodo Nacobbus aberrans (Thorne,1935), Thorne \& Allen 1944
}

\author{
Oscar Castiblanco ${ }^{1}$, Javier Franco ${ }^{2}$, Ramiro Montecinos ${ }^{2}$
}

\begin{abstract}
Resumen
Por pruebas de invernadero y laboratorio se estudió la variabilidad patogénica de nueve poblaciones de Nacobbus aberrans provenientes de diferentes localidades de Argentina, Bolivia, Ecuador y Perú. Las susceptibilidades de las especies de plantas se evaluaron por la presencia o ausencia de nódulos y/o los diferentes estados de desarrollo de $N$. aberrans presentes en sus raíces. Luego de seleccionar Solanum tuberosum ssp. andigena, Lycopersicum esculentum, Beta vulgaris y Capsicum frutescens como plantas diferenciales, se estableció la existencia de cinco poblaciones patogénicamente diferentes o razas. De estas cinco razas, tres estarían presentes en Bolivia.

Por otro lado, se determinó como hospedantes de $N$. aberrans a veinte especies vegetales entre plantas cultivadas y no cultivadas, colectadas en campos agrícolas de la Estación Experimental de Toralapa y sus alrededores (Cochabamba, Bolivia). Asimismo ocho especies se mostraron como posibles hospedantes por la presencia de J2, J3 y J4 en sus raíces no así trece especies que se comportaron como no hospedantes.
\end{abstract}

Palabras claves adicionales: "Rosario de la papa", razas, rango de hospedantes, Bolivia.

Aceptado para publicación: setiembre 1998.

1 Parte de tesis para obtener el título de Técnico Superior Agrónomo, Cochabamba, Bolivia.

2 Departamento de Hematología, Programa de Investigación de la Papa (PROINPA), Casilla 4285, Cochabamba, Bolivia. 


\title{
Races and Range of Plant Host Species in Different Populations of Nacobbus aberrans (Thorne 1935), Thorne and Allen 1944
}

\begin{abstract}
Summary
The variability in pathogenicity of nine populations of Nacobbus aberrans, from different Latín American locations of Argentina, Bolivia, Ecuador and Perú was established by greenhouse and iaboratory evaluations. Plant species were evaluated for presence or absence of root nodules and $N$. aberrans developing stages in the roots.
\end{abstract}

After selecting S. tuberosum ssp. andigena, L. esculentum, B. vulgaris and $C$. frutescens as differential plants, five different strains of $N$. aberrans were identified and three of them are present in Bolivia.

Besides, among the forty one cultivated and non cultivated plant species collected from fields located in Toralapa Experimental Station and surrounding farmer fields (Cochabamba, Bolivia), twenty behaved as host plants for $N$. aberrans, eight as possible hosts because of the presence of J2. J3 and $\mathrm{J} 4$ in their roots, and thirteen as non-hosts.

Additional índex words:

Potato rosary nematode, strains, host range, Bolivia.

\section{Introducción}

La papa (Solanum tuberosum L.) ocupa un lugar privilegiado en cuanto se refiere a producción, consumo y valor nutritivo en Bolivia.

Los principales problemas biofísicos de la papa, en las distintas zonas agroecológicas donde se produce, son la mala calidad de semillas, plagas, enfermedades, nematodos, deficiente e inadecuado uso de fertilizantes. 
Dentro de estos problemas los nematodos Nacobbus aberrans y Globodera spp. causan grandes pérdidas en la producción de papa (10).

Nacobbus aberrans causante del rosario de la papa está considerado como uno de los mayores obstáculos que limitan cuantitativa y cualitativamente los rendimientos en la producción de papa en la región Andina de Bolivia y Perú (6).

Por otro lado, el incremento de la población y el daño causado por $N$. aberrans dependen de varios factores y quizás el más importante sea el hospedante. Así tenemos que aun cuando algunas plantas son muy sensibles, otras toleran la presencia de altas densidades poblacionales (11).

En Bolivia, una de las malezas hospedantes más favorables a $N$. aberrans es Spergula arvensis (1), y la papa es uno de los hospedantes comerciales más importantes en Sudamérica, sobre todo en los Andes de Bolivia y Perú.

Esta situación sin embargo, se hace aún más compleja al haberse detectado poblaciones de $N$. aberrans con preferencias importantes en cuanto a hospedantes y otras características biológicas en común, que han permitido su separación en razas biológicas o fisiológicas pero bajo diferentes esquemas $(3,7,14)$. Las razas fisiológicas o biológicas son patotipos que pueden ser identificadas por su habilidad para multiplicarse en plantas hospedantes llamadas diferenciales, las cuales difieren en su eficiencia como hospedantes (cultivos o géneros diferentes) 0 tienen diferentes genes de resistencia (dentro de un cultivo o género) (8).

La primera evidencia de una variación fisiológica intraespecífica de $N$. aberrans es probablemente la hallada por Thorne \& Schuster (13), quienes citan algunas plantas en las cuales $N$. serendipiticus y $N$. batatiformis tuvieron diferentes reacciones. La segunda especie se reprodujo en Brassica oleraceae, var. capitata, Brassica rapa, Cucumis sativus L., y Pisum sativum, mientras $N$. serendipiticus no lo hizo. Ahora estas dos especies son consideradas como un complejo de poblaciones de $N$. aberrans (12).

La importancia de este estudio radica en primer lugar en identificar por medio de la multiplicación o capacidad de desarrollo de diferentes poblaciones de $N$. aberrans, en ciertas especies cultivadas que puedan ser empleadas como diferenciales, la presencia de razas.

Por otro lado, de acuerdo a la respuesta que muestren las especies cultivadas y no cultivadas colectadas en campos agrícolas a una población de $N$. aberrans (Toralapa), se identificarán aquellas que se comporten como hospedantes y que estarían permitiendo la sobrevivencia y multiplicación de éste fitoparásito (5). 
El estudio comprendió la ejecución de dos ensayos con la finalidad de determinar en primer lugar, aquellos hospedantes que puedan ser empleados como diferenciales para identificar la presencia de razas de $N$. aberrans y luego identificar nuevos hospedantes de $N$. aberrans en especies cultivadas y no cultivadas.

\section{Materiales y Métodos}

El presente estudio se realizó bajo condiciones de invernadero y laboratorio ubicados en la Estación Experimental Toralapa (3,470 msnm, Cochabamba, Bolivia).

1. Determinación de hospedantes diferenciales. Se utilizaron macetas de 250 cc de capacidad en las que se incorporó suelo estéril en sus 3/4 partes, y luego se realizó la siembra de semillas vegetativas de papa cv Waych'a (Solanum tuberosum ssp. andigena), y semilla botánica de remolacha var. "Rubra" (Beta vulgaris), tomate "Angela Gigante" (Lycopersicum esculentum), pimiento agronómico G-10 (Capsicum annum), quinua (Chenopodium quinoa), ají (Capsicum frutescens) y zanahoria (Daucus carota). Posteriormente a la siembra se adicionó la parte faltante con suelo infestado por $N$. aberrans provenientes de Bolivia: Chuquisaca (Sauce), Cochabamba (Pocoata, Toralapa), La Paz (Patacamaya) y Potosí (Chinoli); Perú: Puno (Puno) y Ancash (Carhuaz); Argentina: Tucumán (Tafí del Valle) y Ecuador: Pichincha (Guayallabamba).

Todo el material fue sembrado con tres repeticiones por especie cultivada y por población de $N$. aberrans.

2. Estudio de la gama de hospedantes. Cuarenta y una especies, tanto de plantas cultivadas y no cultivadas colectadas de campos con papa de la Estación Experimental Toralapa y sus alrededores, así como semillas botánicas y vegetativas de otras especies fueron sembradas en macetas de $250 \mathrm{cc}$ de capacidad. 
La inoculación se realizó con suelo infestado por $N$. aberrans colectado en la Estación Experimental Toralapa, en forma similar al primer caso.

Después de 90 y 94 días de la inoculación en ambos ensayos respectivamente, las plantas fueron evaluadas mediante su extracción y calificación por presencia 0 ausencia de nódulos radiculares mediante una escala modificada por Franco $(2,9)$ que considera por un lado las reacciones de resistencia y susceptibilidad de las plantas como comportamientos dentro de un cultivo (ej. papa) y por otro la reacción de diferentes especies de plantas en relación a su comportamiento como hospedante o no al nematodo (Tabla 1).

Posteriormente a la observación visual, se realizó el análisis en laboratorio, tanto para el primer ensayo como para el segundo, en plantas con síntomas y sin síntomas con la finalidad de determinar si había ocurrido penetración y/o multiplicación de N. aberrans. Para la extracción de $N$. aberrans de las raíces de las plantas cultivadas y no cultivadas se utilizó el método de la licuadora (4).

Tabla 1. Escala para evaluar especies vegetales de acuerdo a la resistencia o eficiencia como hospedantes de N. aberrans.

\begin{tabular}{cccc}
\hline Escala & No. de nódulos & Resistencia 1) & $\begin{array}{c}\text { Eficiencia del } \\
\text { hospedante 2) }\end{array}$ \\
\hline 0 & 0 & $\mathrm{R}$ & NE \\
1 & $1-10$ & PR & PE \\
2 & $11-30$ & PS & ME \\
3 & $31-75$ & S & E \\
4 & $>75$ & AS & AE
\end{tabular}

1) Se utiliza dentro de un cultivo (Resistencia o Susceptibilidad) R=Resistente; PR= Parcialmente resistente; $P S=$ Parcialmente susceptible; $S=$ Susceptible; $A S=$ Altamente susceptible.

2) Se utiliza entre cultivos (Eficiente o no eficiente): $\mathrm{NE}=$ No eficiente; $\mathrm{PE}=\mathrm{Poco}$ eficiente; $\mathrm{ME}=$ Moderadamente eficiente; $E=$ Eficiente; $A E=$ Altamente eficiente.

\section{Resultados y Discusión}

En base a las evaluaciones realizadas en invernadero y en laboratorio se obtuvieron los resultados que se discuten a continuación para cada uno de los ensayos. 


\section{Determinación de razas de Nacobbus aberrans}

En la Tabla 2 se muestran los resultados de comportamiento obtenidos de acuerdo a su eficiencia como hospedantes a las diversas poblaciones de $N$. aberrans de los cultivos probados como posibles diferenciales

Tabla 2. Cultivos evaluados por su eficiencia como hospedantes a diversas poblaciones de Nacobbus aberrans.

\begin{tabular}{|l|ccccccc|}
\hline \multirow{2}{*}{ Población } & \multicolumn{7}{|c|}{ Cultivo } \\
\cline { 2 - 8 } & Papa & Tomate & Remolacha & Beterraga & Quinua & Ají & Zanahoria \\
\hline Patacamaya & $E^{*}$ & PE & ME & ME & PE & PE & NE \\
Sauce Mayu & E & E & E & E & NE & NE & NE \\
Chinoli & E & ME & ME & ME & NE & NE & NE \\
Toralapa & E & E & E & E & NE & NE & NE \\
Puno & E & E & NE & NE & NE & NE & NE \\
Carhuaz & E & E & NE & NE & NE & NE & NE \\
Pocoata & E & NE & NE & NE & NE & NE & NE \\
Tafi del Valle & E & NE & NE & NE & NE & NE & NE \\
Guayallabamba & NE & NE & E & NE & NE & E & ME \\
\hline
\end{tabular}

* $P E=$ Poco eficiente; $M E=$ Moderadamente eficiente; $E=$ Eficiente; NE= No eficiente.

Por el comportamiento observado en las especies vegetales empleadas en relación a las diversas poblaciones se propone a los cultivos Solanum tuberosum ssp. andigena cv. Waych'a (papa), Lycopersicum esculentum (tomate), Beta vulgaris (beterraga) y Capsicum frutescens (ají) como diferenciales para la identificación de razas de $N$. aberrans.

Tomando en cuenta la reacción de los cultivos propuestos como diferenciales a las poblaciones provenientes de los diferentes lugares se establece la existencia de cinco razas (Tabla 3).

Tabla 3. Esquema para la identificación de razas de N. aberrans por la reacción (nódulos) y/o multiplicación específica en cuatro plantas diferenciales.

\begin{tabular}{|lcccc|}
\hline Raza & Papa & Tomate & Beterraga & Ají \\
\hline Raza 1 & $(+)^{*}$ & $(+)$ & $(+)$ & $(+)$ \\
Raza 2 & $(+)$ & $(+)$ & $(+)$ & $(-)$ \\
Raza 3 & $(+)$ & $(+)$ & $(-)$ & $(-)$ \\
Raza 4 & $(+)$ & $(-)$ & $(-)$ & $(-)$ \\
Raza 5 & $(-)$ & $(-)$ & $(-)$ & $(+)$ \\
\hline
\end{tabular}

$(+)=$ Presencia de nódulos o estados adultos y huevos de $N$. aberrans en raíces. $(-)$ = Ausencia de nódulos o estados adultos y huevos de $N$. aberrans en raíces. 
Por las reacciones en estos hospedantes diferenciales propuestos, las poblaciones provenientes de Bolivia: La Paz (Patacamaya), Cochabamba (Pocoata, Toralapa), Chuquisaca (Sauce Mayu) y Potosí (Chinoli); Perú: Puno (Puno) y Ancash (Carhuaz); Argentina: Tucumán (Tafí del Valle); Ecuador: Pichincha (Guayallabamba), se separan en cinco posibles razas (Tabla 4)

Raza 1. Comprende únicamente a la población de Patacamaya (La Paz) que desarrolló en todos los cultivos propuestos como diferenciales.

Raza 2. Reúne a las poblaciones de Chinoli (Potosí), Sauce (Chuquisaca) y Toralapa (Cochabamba) que desarrollaron en los cultivos de papa, tomate, beterraga y no así en ají que le sirve para su identificación.

Raza 3. Incluye a poblaciones de Puno y Carhuaz (Perú), que parasitaron a papa y tomate; a diferencia de las poblaciones 1 y 2, estas no desarrollan en beterraga.

Raza 4. Agrupa a las poblaciones de Pocoata (Cochabamba) y Tafí del Valle (Argentina) que sólo desarrollan en papa; a diferencia de las otras tres razas ya mencionadas, esta población no desarrolla en tomate.

Raza 5. Representada únicamente por la población de Guayallabamba (Ecuador), la cual sólo parásita en ají; a diferencia de las anteriores poblaciones (razas) es la única que no parásita en papa.

De estas cinco razas, en Bolivia estarían presentes la raza 1 (Patacamaya), raza 2 (Chinoli, Sauce y Toralapa) y raza 4 (Pocoata).

Tabla 4. Razas de Nacobbus aberrans en poblaciones de Argentina, Bolivia, Ecuador y Perú.

\begin{tabular}{lcccc}
\hline & \multicolumn{4}{c}{ Diferenciales } \\
Raza & Papa & Tomate & Beterraga & Ají \\
\hline Raza 1(Patacamaya) & $(+)$ & $(+)$ & $(+)$ & $(+)$ \\
Raza 2 (Chinoli, Sauce Mayu, Toralapa) & $(+)$ & $(+)$ & $(+)$ & $(-)$ \\
Raza 3 (Puno, Carhuaz) & $(+)$ & $(+)$ & $(-)$ & $(-)$ \\
Raza 4 (Pocoata, Tafí del Valle) & $(+)$ & $(-)$ & $(-)$ & $(-)$ \\
Raza 5 (Guayallabamba) & $(-)$ & $(-)$ & $(-)$ & $(+)$ \\
\hline
\end{tabular}




\section{Determinación de hospedantes de Nacobbus aberrans}

Los hospedantes de $N$. aberrans se determinaron en 41 especies vegetales cultivadas y no cultivadas.

Entre las plantas recolectadas en campos de papa se determinó una amplia gama de hospedantes alternantes a $N$. aberrans en especies cultivadas y no cultivadas. Estas mostraron un comportamiento variable con respecto al desarrollo y multiplicación del nematodo.

En este estudio muchas raíces de las plantas cultivadas y no cultivadas no nodularon (asintomáticas), pero al ser procesadas en laboratorio, muchas presentaron estadios juveniles, huevos y hembras vermiformes de $N$. aberrans.

La causa de la reacción asintomática se debería por un lado a las bajas temperaturas que se registraron en el presente estudio (promedio de $15^{\circ} \mathrm{C}$ ) y que habrían estimulado al parásito, hacia un estado de quiescencia o evitando la producción de huevos y sobre todo retardando el desarrollo de los estadios juveniles. Por otro lado, es también posible que ciertas plantas sean invadidas por $N$. aberrans, pero no fue posible su alimentación y desarrollo a estados adultos, y/o formen nódulos, manteniéndose en un estado de pseudoparasitismo (transitorio). Es así que de acuerdo, a la ocurrencia de invasión, desarrollo y multiplicación de la población de $N$. aberrans proveniente de Toralapa en las diversas especies vegetales probadas, se definió el comportamiento variable de estas en relación a los criterios establecidos para determinar su eficiencia como hospedantes, tal como se indica a continuación.

Hospedantes eficientes (+): Comprende aquellas 20 especies que en mayor o menor número mostraron la presencia de nódulos radicales (Muy eficientes, eficientes, moderadamente y poco eficientes) y/o presencia de estados adultos de $\mathrm{N}$. aberrans, que indican su normal desarrollo (hembras, machos y huevos), pero en algunos casos con reacción asintomática.

Posibles hospedanes (*): Estas ocho especies permitieron el desarrollo de $N$. aberrans sólo hasta determinados estados (J3 o J4), pero no ocurrió multiplicación (ausencia de huevos).

Plantas no hospedantes (-): En estas 13 especies no ocurrió invasión, por lo que no mostraron ningún estado de desarrollo de $N$. aberrans.

Aplicados estos criterios de comportamiento a las plantas recolectadas, se identificaron como plantas hospedantes (+), no hospedantes (-) y posibles hospedantes $(*)$ a las especies vegetales de las familias que se indican en la Tabla 5. 
Sin embargo, el comportamiento de aquellas especies vegetales consideradas como posibles hospedantes $\left(^{*}\right)$ se viene estudiando bajo temperaturas óptimas, para determinar si en estas plantas invadidas por $\mathrm{J} 2$ y hembras vermiformes que no muestran nodulación, se cumple el ciclo biológico de $N$. aberrans. 
Tabla $5 . \quad$ Comportamiento de diversas especies vegetales a una población aislada de N. aberrans (Toralapa).

\begin{tabular}{|c|c|c|c|c|c|c|}
\hline \multirow[t]{2}{*}{ Familia-Especie } & \multirow[t]{2}{*}{ Nombre vulgar } & \multicolumn{2}{|c|}{ Hospedante (+) } & \multicolumn{2}{|c|}{ Posible $\left(^{*}\right)$} & \multirow{2}{*}{$\begin{array}{c}\text { No hospedante (-) } \\
\text { (No invasión) }\end{array}$} \\
\hline & & (Nod.) & (Hvos) & $(\mathrm{J} 4)$ & $(\mathrm{J} 2, \mathrm{~J} 3)$ & \\
\hline $\begin{array}{l}\text { COMPOSITACEAE } \\
\text { Viguiera mandoni } \\
\text { Cenecio clivicolís } \\
\text { Bidens pilosa } \\
\text { Nierembergia spp. } \\
\text { Tagetes mandoni } \\
\text { Gamochoeta americana } \\
\text { Sonchus asper } \\
\text { Taraxacum ofícinalis } \\
\text { Cosmos bipinathus } \\
\text { Senecio vulgaris }\end{array}$ & $\begin{array}{l}\text { Sunchu } \\
\text { Haych'a } \\
\text { Muñí } \\
\text { K'ipu } \\
\text { anis-anis } \\
\text { Wira-wira } \\
\text { Diente de león } \\
\text { Cosmos }\end{array}$ & $\begin{array}{l}(+) \\
(+)\end{array}$ & $(+)$ & $\begin{array}{l}(*) \\
(*)\end{array}$ & $\left({ }^{\star}\right)$ & $(-)$ \\
\hline $\begin{array}{l}\text { MALVACEAE } \\
\text { Sida ssp } \\
\text { Malva paviflora } \\
\end{array}$ & $\begin{array}{l}\text { P'uka jora } \\
\text { Malva }\end{array}$ & & $(+)$ & & & $(-)$ \\
\hline $\begin{array}{l}\text { LEGUMINOSACEAE } \\
\text { Vicia faba } \\
\text { Vicia spp. }\end{array}$ & $\begin{array}{l}\text { Haba } \\
\text { Vicia }\end{array}$ & & & & & $\begin{array}{l}(-) \\
(-)\end{array}$ \\
\hline $\begin{array}{l}\text { CRUCIFERACEAE } \\
\text { Brassica campestris } \\
\text { Sesvania spp } \\
\text { Capsella bursa pastoris }\end{array}$ & $\begin{array}{l}\text { Nabo silvestre } \\
\text { K'uchi } \\
\text { Bolsa de pastor }\end{array}$ & & $\begin{array}{l}(+) \\
(+)\end{array}$ & & $\left({ }^{\star}\right)$ & \\
\hline $\begin{array}{l}\text { CARYOPHYLLACEAE } \\
\text { Spergula arvensis } \\
\text { Cerastium soratense }\end{array}$ & $\begin{array}{l}\text { Abrojito } \\
\text { Lisa }\end{array}$ & $\begin{array}{l}(+) \\
(+)\end{array}$ & & & & \\
\hline $\begin{array}{l}\text { CHENOPODIACEAE } \\
\text { Chenopodium ambrosioides } \\
\text { Chenopodium murale } \\
\text { Chenopodium albus } \\
\text { Chenopodium quinoa }\end{array}$ & $\begin{array}{l}\text { Paico } \\
\text { Ojara } \\
\text { Quinua silvestre } \\
\text { Quinua } \\
\end{array}$ & $(+)$ & $\begin{array}{l}(+) \\
(+)\end{array}$ & $\left(^{*}\right)$ & & \\
\hline $\begin{array}{l}\text { POACEAE } \\
\text { Hordeum vulgare } \\
\text { Bromus erectus } \\
\text { Eragrostis minor } \\
\text { Avena sativa } \\
\text { Triticum sativum }\end{array}$ & $\begin{array}{l}\text { Cebada } \\
\text { Paja } \\
\text { Avena } \\
\text { Trigo } \\
\end{array}$ & & & $\left({ }^{*}\right)$ & & $\begin{array}{l}(-) \\
(-) \\
(-) \\
(-)\end{array}$ \\
\hline \begin{tabular}{|l|} 
DIPSACACEAE \\
Scabiosa columbaha
\end{tabular} & Espuela & & & & & $(-)$ \\
\hline $\begin{array}{l}\text { POLIGONACEAE } \\
\text { Poligonium laccrum }\end{array}$ & Duraznillo & & & & $\left({ }^{\star}\right)$ & \\
\hline $\begin{array}{l}\text { UMBELIFLORAE } \\
\text { Erodium cicutarium }\end{array}$ & Reloj reloj & $(+)$ & & & & \\
\hline $\begin{array}{l}\text { PINOIDEAS } \\
\text { Pinnus radíata }\end{array}$ & Pino & & & & & $(-)$ \\
\hline $\begin{array}{l}\text { CUPRESACEAE } \\
\text { Cupressus macrocarpa }\end{array}$ & Ciprés & & & & & $(-)$ \\
\hline $\begin{array}{l}\text { MIMOSIODEAS } \\
\text { Acacia horrida }\end{array}$ & Acacia & & & & & $(-)$ \\
\hline $\begin{array}{l}\text { ESCROPHULARIACEAE } \\
\text { Antirrinum majus }\end{array}$ & & $(+)$ & & & & \\
\hline $\begin{array}{l}\text { MIRTACEAE } \\
\text { Eucaliptus globulus }\end{array}$ & Eucalipto & & & & & $(-)$ \\
\hline $\begin{array}{l}\text { SOLANACEAE } \\
\text { Solanum spp } \\
\text { Lycopersicon esculentum }\end{array}$ & $\begin{array}{l}\text { Papa } \\
\text { Tomate }\end{array}$ & $\begin{array}{l}(+) \\
(+)\end{array}$ & & & & \\
\hline $\begin{array}{l}\text { OXALIDACEAE } \\
\text { Oxalis acetocella } \\
\text { Oxalis spp. } \\
\text { Oxalis tuberosa }\end{array}$ & $\begin{array}{l}\text { K'ellu tica } \\
\text { Oca }\end{array}$ & $\begin{array}{l}(+) \\
(+)\end{array}$ & $(+)$ & & & \\
\hline
\end{tabular}




\section{Referencias Bibliográficas}

1. Caero, G. 1985. Estudios realizados y actividades dentro de la investigación nematológica en Bolivia. En: Investigaciones Nematológicas en Programas Latinoamericanos de Papa. J. Franco y H. Rincón (eds.). Centro Internacional de la Papa (CIP). 41-46.

2. Castiblanco, O. 1992. Razas y gama de hospedantes en diferentes poblaciones de Nacobbus aberrans. Tesis Técnico Superior en Agronomía, Universidad Mayor de San Simón. Cochabamba, Bolivia. 73 p.

3. Centro Internacional de la Papa (CIP). 1992. Annual Report CIP 1992. Lima, Perú. 254 p.

4. Costilla, M. 1985. El nematodo del rosario o el falso nematodo del nodulo Nacobbus aberrans (Thorne, 1935) Thorne \& Allen, 1944 y su relación con el cultivo de papa en el Noroeste Argentino. En: Investigaciones Nematológicas en Programas Latinoamericanos de papa. J. Franco y H. Rincón (eds.). Centro Internacional de la Papa (CIP): 3-16.

5. Costilla, M. 1985. Grado de susceptibilidad y resistencia de plantas cultivadas y no cultivadas al falso nematodo del nodulo Nacobbus aberrans (Thorne, 1935) Thorne \& Allen 1944 en la Argentina. En: Investigaciones Nematológicas en Programas Latinoamericanos de Papa. J. Franco y H. Rincón (eds.). Centro Internacional de la Papa (CIP): 21-24.

6. Franco, J. 1994. Problemas de nematodos en la producción de papa en climas templados en la región andina. Nematrópica 24: 179-195.

7. Inserra, R.; G. Griffin and J. Anderson. 1985. The false root-knot nematode Nacobbus aberrans. Utah Agric. Exp. Sta. Research Bulletin 510: 14 p.

8. Programa de Investigación de la Papa (PROINPA). 1991. Informe Anual 1990-91. Cochabamba, Bolivia. 260 p.

9. Programa de Investigación de la Papa (PROINPA). 1992. Informe Anual 1991-92. Cochabamba, Bolivia. 297 p.

10. Ramos, J.; J. Franco; N. Ortuño; R. Oros y G. Maín. 1998. Incidencia y severidad de Nacobbus aberrans y Globodera spp. en el cultivo de la papa en Bolivia: Pérdidas en el valor bruto de su producción. Documento de trabajo No. 7., Programa de Investigación de la Papa. Cochabamba, Bolivia. 201 p. 
11. Sasser, J. N. 1989. Plant Parasitic Nematodes; The Farmer's Hidden Enemy. Department of Plant Pathology and Consortium for International Crop Protection, Raleigh. NC. $115 p$.

12. Sher, S. A. 1970. Revisión of the genus Nacobbus Thorne and Allen, 1944 (Nematodo: Tylenchoidea). Journal of Nematology 2: 228-235.

13. Thorne, G. and L. M. Schuster. 1956. Nacobbus bataliformis n. sp. (Nematodo: Tylenchidae) producing galls on roots of sugar-beets and other plants. Proc. of the Helm. Soc. of Washington. 23:128-134.

14. Toledo, R., C. Sosa-Moss y E. Zabaleta. 1993. Características patogénicas de cinco poblaciones de Nacobbus aberrans. Nematrópica 23: 105-108. 